\title{
Malignant fibrous histiocytoma of the pulmonary vein with prolapse through the mitral valve orifice
}

\author{
Miqdad Ali Khan and Robert C. Davidson
}

Michigan State University, Blodgett Memorial Medical Center, Grand Rapids, Michigan 49506, USA

\begin{abstract}
Summary: We present the case of a 33 year old white woman with malignant fibrous histiocytoma arising from the pulmonary vein and prolapsing through the mitral valve, causing acute heart failure. The patient underwent emergency cardiothoracic surgery with resection of the tumour while on cardiopulmonary bypass. The patient's heart failure improved immediately following the resection, demonstrating the benefit of palliative surgery. Adjuvant chemotherapy was recommended and the patient did well for 18 months of follow-up.
\end{abstract}

\section{Introduction}

Malignant fibrous histiocytoma (MFH) is usually a soft tissue tumour. It represents $10 \%$ of all soft tissue sarcomas. The most common site of origin of this tumour is the extremities or retroperitoneum. On a survey of the literature we could find no previous report of MFH arising from the pulmonary vein. There have been reports, however, of pulmonary artery involvement. ${ }^{1,2}$

\section{Case report}

A 33 year old white woman was in good health until the sudden onset of sharp, pleuritic substernal pain. The pain persisted for 24 hours prior to admission and was associated with shortness of breath which was worse when leaning forward or lying down. She had noted a mass growing in her right scapular region during the 3 weeks prior to admission. There was no history of weight loss or any other constitutional symptoms.

Physical examination on admission revealed a supine blood pressure of $122 / 174 \mathrm{mmHg}$ with pulse rate of 104 per minute, and sitting blood pressure of $124 / 82 \mathrm{mmHg}$ with pulse rate of 104 per minute. Her respiratory rate was 20 per minute. She had bilateral basal râles with no dependent oedema. A $2 \mathrm{~cm}$ small mobile soft tissue mass was noted overlying the right scapular region. The rest of the physical examination was unremarkable, including normal pelvic examination.

Correspondence: Miqdad Ali Khan, M.D., Sinai Samaritan Medical Centre, Winter Research Building 427, 945N 12 St, PO Box 342, Milwaukee, WI 53201-0342, USA.

Accepted: 5 February 1992
The laboratory evaluation showed haematocrit of 31.6 , haemoglobin of $11.0 \mathrm{mg} / \mathrm{dl}$ with normocytic and normochromic indices. Her cardiac isoenzymes were within normal limits and her chest $\mathrm{X}$-ray showed a left lower lobe infiltrate associated with left pleural effusion. Her electrocardiogram had non-specific $T$-wave changes in the anterior precordial leads and some ST sagging in the inferior leads.

An excisional biospy of the soft tissue from the right scapular region showed MFH on histological examination. Echocardiograph (M-mode and twodimensional) demonstrated a large left atrial mass that was prolapsing into the left ventricle from the left atrium and causing obstruction of the mitral orifice (Figures 1 and 2). It did not appear to be arising from the inter-atrial septum and was thought to be entering the left atrium from the pulmonary vein (Figure 3 ). Doppler study revealed mild mitral regurgitation. The left atrial mass was also seen on the computed tomographic (CT) scan of the chest.

On the fourth hospitalization day, the patient developed sudden haemoptysis and her blood pressure dropped to $80 / 50 \mathrm{mmHg}$ with signs of congestive heart failure. Emergency surgery was performed in the belief that the cardiac mass was obstructing the mitral orifice causing her cardiovascular decompensation. Her pulmonary artery diastolic pressure was noted to be $18 \mathrm{mmHg}$ and central venous pressure $7 \mathrm{mmHg}$. While on cardiopulmonary bypass, a large $8 \mathrm{~cm}$ tumour occupying the left atrium arising from the orifice of the left inferior pulmonary vein was found and excised. There was some evidence of traumatic injury of the posterior leaflet of the mitral valve by the mass. Histological examination revealed that both of the tumours (right scapular region and the pulmonary 


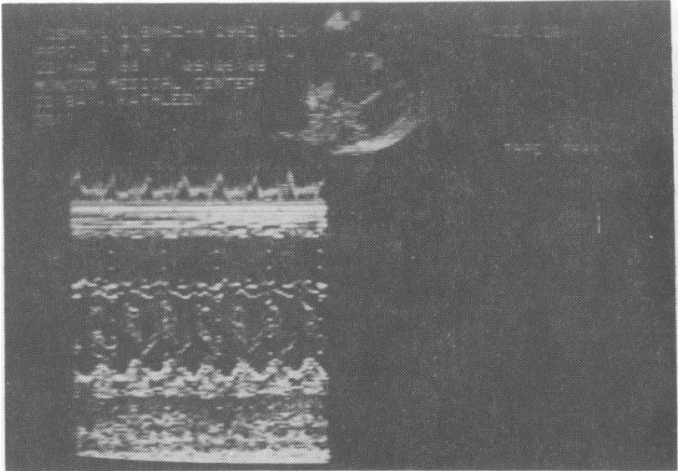

Figure 1 M-mode echocardiogram through the mitral valve showing a mass prolapsing through the mitral orifice in diastole.

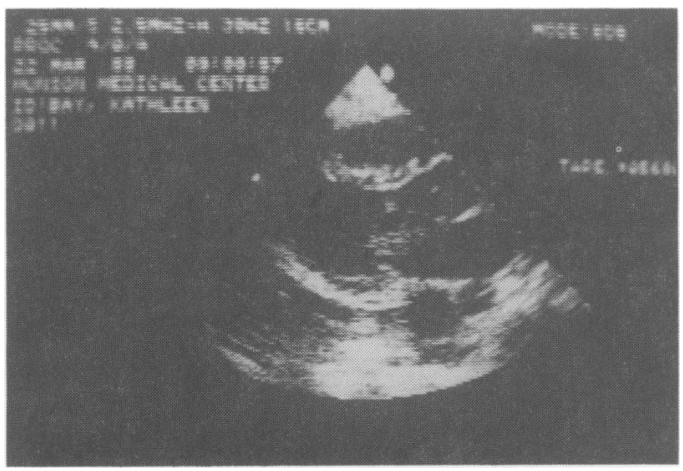

Figure 2 Para-sternal long axis view demonstrating mitral valve in diastole with a large mass prolapsing through the mitral orifice.

vein) were of similar type, having spindle cells with storiform appearance, atypical mitotic figures and abundant nuclear pleomorphism.

The patient did well haemodynamically after the surgery and did not require fluid or vasopressor agents after the resection of the tumour. She was discharged from the hospital on the fifth postoperative day. Although she now has metastatic disease after 18 months, there is no clinical or echocardiographic evidence of recurrent left atrial mass.

\section{Discussion}

We report this case not only because malignant fibrous histiocyctoma originating from the pulmonary vein is previously unrecorded, prolapsing through the mitral valve and obstructing the mitral valve orifice, but also the patient's excellent response to palliative surgery. The primary site of the

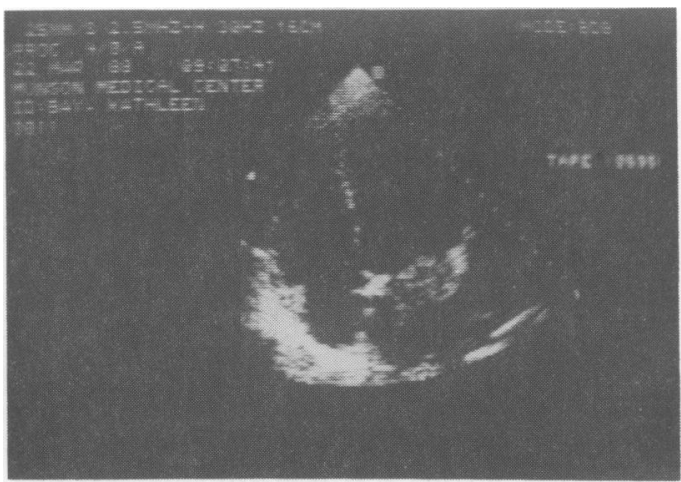

Figure 3 Apical four chamber view demonstrates mass in the left atrium during ventricular systole. Of note is a clear lack of attachment of the tumour to the inter-atrial septum.

tumour was most likely from the pulmonary vein rather than the soft tissue overlying the scapula for two reasons: (a) the lack of demonstrable pulmonary metastases which would be expected if the haematogenous metastases occurred from the peripheral site; and (b) the much larger size of the pulmonary vein mass as compared to the scapular region mass. In regards to the former, Weiss noted in his analysis of 200 cases that metastases usually occurred within 2 years after the initial diagnosis and were primarily through the haematogenous route, frequently to the lungs $(82 \%)$, liver and bone $(18 \%){ }^{3}$ On the contrary, one would not expect pulmonary metastases from a lesion originating in the left heart, since the haematogenous distribution would be to the systemic circulation (that is, right scapular region) rather than to the pulmonary vasculature.

MFH of thoracic origin involving lungs, mediastinum and pericardium is rare, but is being reported more often recently because of better classification and differentiation of this entity. Large vessel sarcomas are more common in the veins $(75 \%)$ with $50 \%$ occurring in the inferior vena cava. Involvement of the pulmonary arteries is twice as common as of systemic arteries. ${ }^{4}$ As the name implies these are malignant tumours with local recurrence as well as metastases (in about half of the patients). ${ }^{3,5,6}$

The five-year survival rate for MFH has been reported to be $50 \% .^{5}$ Kearney found five-year survival to be $28 \%$ in his retrospective study with proximally located tumours compared to the distally located tumours having a survival rate of $73 \% .^{6}$ The retroperitoneal tumours had a $14 \%$ survival rate in the same study. Surgical resection is the mainstay of therapy. ${ }^{7}$ An aggressive combined approach utilizing surgery and adjuvant chemotherapy is currently the treatment of choice for the peripheral and truncal sarcomas. ${ }^{8}$ 
Congestive heart failure appears to be the most common presentation in patients with cardiac tumours $^{9}$ as was the case in our patient who decompensated so acutely that emergency cardiac surgery was needed. She showed dramatic clinical improvement after resection of her left atrial mass. We suggest that palliative surgery should be considered for similar patients with cardiac mass who develop haemodynamic instability. Echocardio-

\section{References}

1. Carlin, B.W. \& Moser, K.M. Pulmonary artery obstruction due to malignant fibrous histiocytoma. Chest 1987, 92: 173175.

2. Van Damme, H., Vaneerdeweg, W. \& Schoofs, E. Malignant fibrous histiocytoma of the pulmonary artery. Ann Surg 1987, 205: 203-207.

3. Weiss, S.H. \& Enzinger, F.M. Malignant fibrous histiocytoma Cancer 1978, 41: 2250-2266.

4. Killebrew, E. \& Gerbode, F. Leiomyosarcoma of the pulmonary artery diagnosed preoperatively by angiocardiography. Replacement with a composite graft. J Thorac Cardiovasc Surg 1976, 71: 469-471.

5. Enjoji, M., Hashimoto, H. \& Iwasaki, H. Malignant fibrous histiocytoma: A clinicopathologic study of 130 cases. Acta Pathol Jpn 1980, 30: 727-741. graphy was able to help us in demonstrating a mass arising from the pulmonary vein and entering the left atrium with prolapse through the mitral valve, and MFH should be considered among the differential diagnosis of such lesions. We recommend that echocardiography should be part of the routine investigation whenever malignant fibrous histiocytoma is detected anywhere in the body in patients with signs and symptoms of heart failure.

6. Kearney, M.M., Soule, E.H. \& Ivins, J.C. Malignant fibrous histiocytoma: A retrospective analysis of 167 cases. Cancer 1980, 45: 167-178.

7. Magovern, G.J., Yusuf, M.F., Liebler, G.A., Pugh, R.P. \& Joyner, C.R. The surgical resection and chemotherapy of metastatic osteogenic sarcoma of the right ventricle. Ann Thorac Surg 1980, 29: 76-80.

8. Leite, C., Goodwin, J.W., Sinkovics, J.G., Baker, L.H. \& Benjamin, R. Chemotherapy of malignant fibrous histiocytoma. Cancer 1977, 40: 2010-2014.

9. Hallahan, D.E., Vogelzang, N.J., Borow, K.M., Bostwick, D.G. \& Simon, M.A. Cardiac metastases from soft-tissue sarcomas. J Clin Oncol 1986, 4: 1662-1669. 